\title{
The GS09 double parton distribution functions
}

\author{
Jonathan Gaunt*i \\ University of Cambridge \\ E-mail: gaunt@hep.phy.cam.ac.uk
}

It is anticipated that hard double parton scattering (DPS) will occur frequently in the collisions of the LHC, producing interesting signals and significant backgrounds to certain single scattering processes. In order to make theoretical predictions of double scattering rates and properties, the double parton distributions (dPDFs) $D_{p}^{j_{1} j_{2}}\left(x_{1}, x_{2} ; Q_{A}, Q_{B}\right)$ are required. We discuss the first publicly available set of equal-scale $\left(Q_{A}=Q_{B}\right)$ leading order dPDFs - the GS09 dPDFs. A brief account is given describing how pQCD evolution effects and sum rule constraints (the latter derived by us) have been incorporated into this set of dPDFs. We then present a summary of a phenomenological investigation into same-sign $\mathrm{W}$ pair production conducted using GS09. In this, the DPS signal produced using GS09 is compared with that obtained using simple products of single PDFs $\times\left(1-x_{1}-x_{2}\right)^{n}$, and the single scattering backgrounds $\left(W^{ \pm} W^{ \pm} j j\right.$, di-boson and heavy flavour) are carefully calculated. It is found that the correlations in GS09 manifest themselves in non-trivial kinematic correlations between the $\mathrm{W}$ bosons. However, it is unlikely that these correlations will be measurable at the LHC in the near future owing to the fact that the background is significant even after cuts.

XVIII International Workshop on Deep-Inelastic Scattering and Related Subjects

April 19 -23, 2010

Convitto della Calza, Firenze, Italy

\footnotetext{
* Speaker.

${ }^{\dagger}$ This report summarises work performed in collaboration with C.-H. Kom, A. Kulesza, and W. J. Stirling. JG is supported by the UK Science and Technology Facilities Council.
} 


\section{Introduction}

Double parton scattering (DPS) is said to occur when two pairs of partons participate in hard interactions during a single proton-proton collision. This process is drawn schematically in figure 1, in which all of the variables relevant to DPS have also been defined. Assuming the factorisation of the hard subprocesses $A$ and $B$, and further assuming that the generalised double parton distributions $\Gamma_{i j}\left(x_{1}, x_{2}, b ; Q_{A}, Q_{B}\right)$ may be decomposed into a longitudinal piece and a piece depending only on the transverse separation $b$, we may write the cross section for DPS as follows:

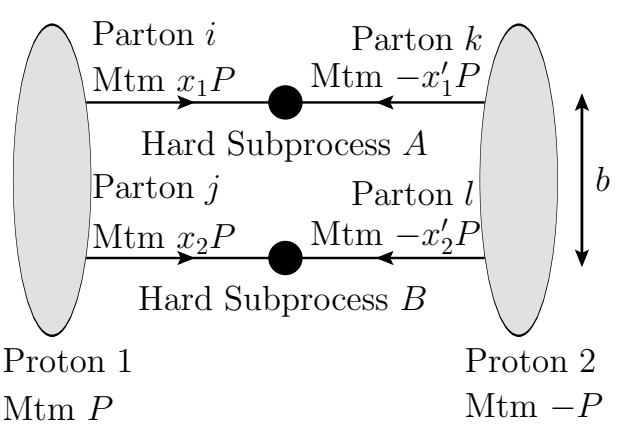

Figure 1: Schematic representation of DPS.

$$
\sigma_{(A, B)}^{D P S}=\frac{m}{2 \sigma_{\mathrm{eff}}} \sum_{i, j, k, l} \int d x_{1} d x_{2} d x_{1}^{\prime} d x_{2}^{\prime} D_{p}^{i j}\left(x_{1}, x_{2} ; Q_{A}, Q_{B}\right) D_{p}^{k l}\left(x_{1}^{\prime}, x_{2}^{\prime} ; Q_{A}, Q_{B}\right) \hat{\sigma}_{i k}^{A}\left(x_{1}, x_{1}^{\prime}\right) \hat{\sigma}_{j l}^{B}\left(x_{2}, x_{2}^{\prime}\right)
$$

The function $D_{p}^{i j}\left(x_{1}, x_{2} ; Q_{A}, Q_{B}\right)$ is known as a double parton distribution function (dPDF) and may be interpreted at $\mathrm{LO}$ as the probability of finding a pair of partons in the proton with flavours $i$ and $j$, longitudinal momentum fractions $x_{1}$ and $x_{2}$, when probed at scales $Q_{A}$ and $Q_{B}$. The quantity $\sigma_{e f f}$ is a non-perturbative factor which is related to the pair density distribution in transverse space. We take it to be a constant and equal to the CDF measured value of $14.5 \mathrm{mb}$, with the caveat that the value of this quantity should be re-established at LHC scales using benchmark processes. $m$ is a symmetry factor that equals 1 if $A=B$ and 2 otherwise, and the $\hat{\sigma}_{i k}^{A}$ are parton-level cross sections.

In many of the extant studies of DPS (see [1] for a complete list of references), a further assumption which has been applied is to take the dPDFs as products of sPDFs (i.e. ignore longitudinal momentum correlations). It is argued that this is justifiable at low $x_{i}$ due to the large population of partons at these $x$ values, and there is some evidence from CDF [2] that it holds for sea partons at low $x$. Under this additional approximation, the DPS cross section reduces to a product of single parton scattering (SPS) cross sections:

$$
\sigma_{(A, B)}^{D P S}=\frac{m}{2} \frac{\sigma_{(A)}^{S} \sigma_{(B)}^{S}}{\sigma_{\text {eff }}}
$$

We recall that the SPS cross section for a given hard process grows with energy as the single PDFs (sPDFs) are probed at lower $x$ values where they are larger. Equation (1.2) demonstrates that the DPS cross section goes approximately as the product of SPS cross sections - therefore DPS cross sections grow faster with energy than SPS ones, and DPS will be more important at the LHC than at any previous collider. It has already been established using (1.2) that DPS processes constitute important backgrounds to Higgs and other interesting signals (see e.g. [3]). In addition to this, DPS can be considered as an interesting signal process in its own right, as it reveals information about the correlations between pairs of partons in the proton.

For these reasons, we need to ensure we have a good theoretical handle on DPS. It has already been established that the form (1.2) is not adequate. In [ [ 4 a LO 'double DGLAP' (dDGLAP) equation was derived describing the scaling violations of the dPDFs with a common factorisation 
scale $\left(Q_{A}=Q_{B} \equiv Q\right)$. A crucial prediction of this is that even if the dPDFs may be taken to be equal to products of sPDFs at one scale, then at any other scale the dPDFs will deviate from factorised forms.

Further to this, simple factorised forms do not obey the following sum rule constraints, which we have shown are preserved by LO dDGLAP evolution if they hold at some starting scale [1]:

Momentum Sum Rule: $\sum_{j_{1}} \int_{0}^{1-x_{2}} d x_{1} x_{1} D_{p}^{j_{1} j_{2}}\left(x_{1}, x_{2} ; Q\right)=\left(1-x_{2}\right) D_{p}^{j_{2}}\left(x_{2} ; Q\right)$

Number Sum Rule: $\quad \int_{0}^{1-x_{2}} d x_{1} D_{p}^{j_{1 v} j_{2}}\left(x_{1}, x_{2} ; Q\right)= \begin{cases}N_{j_{1 v}} D_{p}^{j_{2}}\left(x_{2} ; Q\right) & \text { when } j_{2} \neq j_{1} \text { or } \bar{j}_{1} \\ \left(N_{j_{1 v}}-1\right) D_{p}^{j_{2}}\left(x_{2} ; Q\right) & \text { when } j_{2}=j_{1} \\ \left(N_{j_{1 v}}+1\right) D_{p}^{j_{2}}\left(x_{2} ; Q\right) & \text { when } j_{2}=\bar{j}_{1}\end{cases}$

The symbol $j_{1 v} \equiv j_{1}-\bar{j}_{1}\left(j_{1} \neq g\right)$, and $N_{j_{1 v}}$ is the number of 'valence' $j_{1}$ quarks in the proton. These sum rules have simple interpretations in terms of conditional probabilities. The first states that if you observe a parton with momentum fraction $x_{2}$ in the proton, the momentum fractions of all other partons must add up to $1-x_{2}$. The second states that if you observe a parton with flavour $j$ in the proton, the number of partons of flavour $j$ elsewhere in the proton must be reduced by one (we use the term 'number effects' to describe this simple phenomenon).

In this contribution we discuss the first publicly available set of LO equal-scale dPDFs - the GS09 dPDFs - which incorporates pQCD evolution effects plus the sum rule constraints. Section 2 describes how the dPDFs were constructed incorporating these features [1], whilst section 3 summarises a phenomenological study of DPS performed using GS09 [5].

\section{The GS09 dPDFs}

The GS09 dPDF package comprises a grid of dPDF values spanning the ranges $10^{-6}<x_{1}<1$, $10^{-6}<x_{2}<1,1 \mathrm{GeV}^{2}<Q^{2}<10^{9} \mathrm{GeV}^{2}$, which is available along with interpolation code from HepForge [6]. It has been obtained by constructing inputs that approximately satisfy the sum rules at $Q_{0}=1 \mathrm{GeV}$, and then numerically evolving these inputs up to higher scales according to the dDGLAP equation. The sPDF set to which we have chosen our dPDF set to correspond is (a slightly modified version of) the MSTW2008LO set [7].

Given the paucity of experimental data regarding the dPDFs, and in accordance with simple arguments and the CDF results, we base our inputs on factorised products of MSTW2008LO sPDFs. However, we modify these basic forms in several ways to ensure that the input dPDFs approximately satisfy the sum rules.

First, all of the dPDFs are multiplied by a factor $\rho^{i j}\left(x_{1}, x_{2}\right)$ which is designed to take account of phase space effects. This factor should ensure the appropriate behaviour of the dPDFs near the kinematic boundary $x_{1}+x_{2}=1$ - namely, a smooth decrease to zero. It was discovered that the following form for $\rho^{i j}$ gives rise to inputs which satisfy the momentum sum rules (plus appropriate number sum rules) well:

$$
\rho^{i j}\left(x_{1}, x_{2}\right)=\left(1-x_{1}-x_{2}\right)^{2}\left(1-x_{1}\right)^{-2-\alpha(j)}\left(1-x_{2}\right)^{-2-\alpha(i)}
$$

$\alpha(i)$ is 0 if $i$ is a sea parton, and 0.5 if it is a valence parton. 
We recall that there are only a finite number of valence quarks in the proton, as opposed to an infinite number of sea quarks and gluons. Number effects are therefore most significant in the context of valence quarks, and on this basis we have chosen to only take account of valence number effects in our inputs. This is done by dividing the $u_{v} u_{v}$ part of any dPDF by two, and completely subtracting the $d_{v} d_{v}$ part. The reasoning behind this is that removing one up valence quark essentially halves the probability to find another, whilst there is no chance of finding two valence down quarks in the proton.

Finally, we have added extra terms to input distributions whose flavour indices contain $j \bar{j}$ combinations to take account of so-called ' $j \bar{j}$ correlations'. These are essentially related to sea parton number effects, although they can alternatively be thought of as arising during evolution from some lower scale to $Q_{0}$ via $g \rightarrow j \bar{j}$ splittings. It is important to include these terms in the equal flavour valence-valence $\left(j_{v} j_{v}\right)$ inputs since the ' $j \bar{j}$ correlation' term is much larger for these than the quasi-factorised piece at low $x$.

With these adjustments, our dPDF inputs satisfy all sum rules to better than $25 \%$ accuracy for $x \lesssim 0.8$ (in the normal 'double human' basis).

\section{Effects of using GS09 dPDFs on same-sign WW DPS signal}

It is interesting to ask how the inclusion of proper $\mathrm{pQCD}$ evolution and sum rule constraints in GS09 affects experimentally measurable DPS signals. In [7], the same-sign WW DPS signal produced using GS09 was compared with that arising from simple factorised forms. The factorised forms used were simple products of MSTW2008LO dPDFs multiplied by $\left(1-x_{1}-x_{2}\right)^{n}, n=0,1,2$ (the ' $\mathrm{MSTW}_{n}$ ' dPDFs). Same-sign WW production was chosen as the DPS process because it has been traditionally considered as a clean channel for observation of DPS. The cross section for same-sign WW production via SPS is suppressed to the same order of magnitude as the DPS cross section due to the large number of vertices required in the Feynman diagrams. What is more, this SPS background must always produce two jets in addition to the WW pair - so it can be efficiently removed via a jet veto.

We chose to study the signal in the case when both Ws decay leptonically. A difference in the GS09 and $\mathrm{MSTW}_{n}$ sets can be observed in the following quantity which is particularly sensitive to longitudinal correlations:

$$
a_{\eta_{l}}=\frac{\sigma\left(\eta_{l_{1}} \times \eta_{l_{2}}<0\right)-\sigma\left(\eta_{l_{1}} \times \eta_{l_{2}}>0\right)}{\sigma\left(\eta_{l_{1}} \times \eta_{l_{2}}<0\right)+\sigma\left(\eta_{l_{1}} \times \eta_{l_{2}}>0\right)}
$$

This quantity is plotted as a function of the minimum pseudorapidity cut on the detector hemispheres in figure 2. The GS09 values for $a_{\eta_{l}}$ are larger because the probability of a proton providing two large $x$ (valence) partons is reduced under GS09 (due to number effects), so GS09 results in fewer events with the two leptons in the same hemisphere.

In addition to a comparison of GS09 and factorised forms, [5] examines sources of background aside from the canonical SPS WW processes. It is found that there are several processes that can give rise to the same-sign lepton signal in the detectors:

$$
\begin{array}{llrl}
\text { Heavy flavour: } & g g \rightarrow t \bar{t}, & t \rightarrow W^{+} b \rightarrow l^{+} v b, & \bar{t} \rightarrow W^{-} \bar{b} \rightarrow q \bar{q}^{\prime} l^{+} v \bar{c} \\
& g g \rightarrow b \bar{b} \rightarrow B \bar{B}+\ldots, & B \rightarrow l^{+} v X, & \bar{B}^{0} \rightarrow B^{0} \rightarrow l^{+} v \tilde{X}
\end{array}
$$




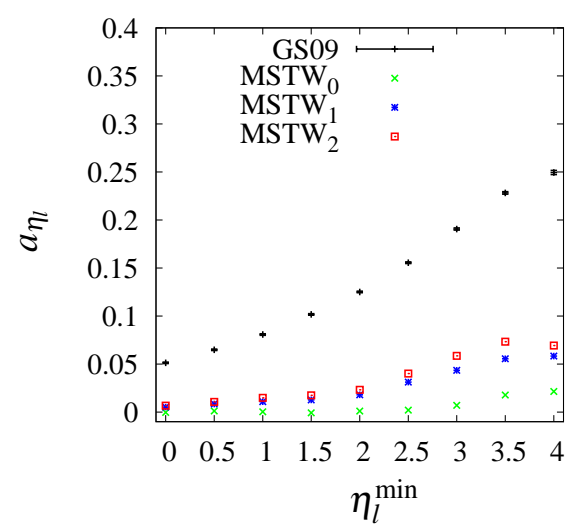

(a) Positively charged leptons

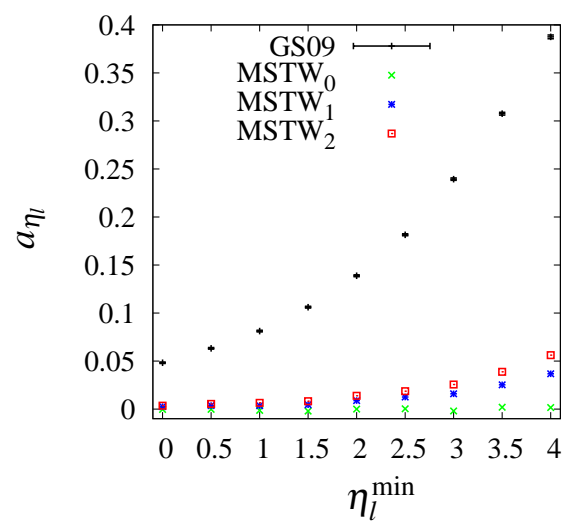

(b) Negatively charged leptons

Figure 2: Pseudorapidity asymmetry $a_{\eta_{l}}$ for $p p$ collisions at $\sqrt{s}=14 \mathrm{TeV}$ evaluated using different dPDFs. No cuts are applied.

$$
\begin{array}{ll}
\text { Electroweak gauge boson pair: } & q \bar{q}^{\prime} \rightarrow W^{+} Z\left(\gamma^{*}\right) \rightarrow l^{+} v l^{+}\left(l^{-}\right) \\
& q \bar{q} \rightarrow Z\left(\gamma^{*}\right) Z\left(\gamma^{*}\right) \rightarrow l^{+}\left(l^{-}\right) l^{+}\left(l^{-}\right)
\end{array}
$$

The leptons in brackets must fall outside the detector acceptance to give rise to the appropriate signal, which in our study meant that they had to satisfy $|\eta|>2.5$. The variety and strength of the backgrounds means that a careful choice of cuts is required to enhance the signal/background as much as possible - these are listed in [5]. Even with these cuts, the backgrounds are nonnegligible and it is unlikely that a conclusive discrimination between GS09 and factorised forms will be possible using this channel in the near future. On the other hand, cleaner channels with regards to DPS may exist - we are currently examining double Drell-Yan as a possibility.

\section{References}

[1] J. R. Gaunt and W. J. Stirling, Double Parton Distributions Incorporating Perturbative QCD Evolution and Momentum and Quark Number Sum Rules, JHEP 03 (2010) 005, [arXiv: 0910.4347].

[2] CDF Collaboration, F. Abe et. al., Double parton scattering in $\bar{p} p$ collisions at $\sqrt{s}=1.8 T e V$, Phys. Rev. D56 (1997) 3811-3832.

[3] A. Del Fabbro and D. Treleani, A double parton scattering background to Higgs boson production at the LHC, Phys. Rev. D61 (2000) 077502, [hep-ph/9911358]

[4] R. Kirschner, Generalized Lipatov-Altarelli-Parisi equations and jet calculus rules, Phys. Lett. $\mathbf{B 8 4}$ (1979) 266; V. P. Shelest, A. M. Snigirev, and G. M. Zinovjev, Gazing into the multiparton distribution equations in QCD, Phys. Lett. B113 (1982) 325.

[5] J. R. Gaunt, C.-H. Kom, A. Kulesza, and W. J. Stirling, Same-sign W pair production as a probe of double parton scattering at the LHC, arXiv:1003.3953.

[6] http://projects.hepforge.org/gsdpdf.

[7] A. D. Martin, W. J. Stirling, R. S. Thorne, and G. Watt, Parton distributions for the LHC, Eur. Phys. J. C63 (2009) 189-285, arXiv: 0901.0002 ]. 